\title{
Assessment of Operant Learning and Memory in Mice Born
}

\section{through Intracytoplasmic Sperm Injection}

17 Matthew Peterson (0000-0001-6437-8609), Linda Hayes (0000-0003-0553-3490), Wei Yan 0682). 


\section{Abstract}

22 Study question: Are there differences in operant learning and memory between mice born through intracytoplasmic sperm injection (ICSI) and naturally-conceived control (CTL) mice?

Summary answer: ICSI females exhibited deficits in acquisition learning relative to CTL

females, whereas ICSI males exhibited deficiency in discrimination learning and memory relative to CTL males during initial assessments. ICSI and CTL groups exhibited equally poor long-term retention of learned discrimination and memory performances at old age.

What is known already: Some human outcome studies have suggested that ICSI might be associated with an increased risk of certain cognitive disorders, but only one of two behavioral studies with ICSI mouse models have reported differences between ICSI and CTL females. No studies to date have investigated associative learning in ICSI mice.

Study design, size, duration: 36 ICSI mice (18 male, 18 female) and 37 CTL mice (19 male, 18 female) aged 3-6 months were compared in a series of operant learning procedures that assessed acquisition of a new behavior, discrimination learning, and memory. 16 ICSI mice (9 male, 7 female) and 17 CTL mice (10 males, 7 females) received follow-up discrimination learning and memory assessments at 12 months of age (six months after the end of initial training) to evaluate retention and reacquisition of learned performances.

Participants/materials, setting, methods: Mice received daily operant learning sessions in experimental chambers in which all stimulus events and the recording of responses were automated. Food rewards were delivered for responding under different conditions of reinforcement, which varied by procedure. Subjects received a successive series of sessions of nose poke acquisition training, discrimination training, and the delayed non-matching-to-position (DNTMP) memory procedure. Mixed repeated measures ANOVAs in which the between- 
subjects factor was group (ICSI vs. CTL) and the within-subjects factor was repeated exposures

to learning procedures (i.e., sessions) were used to analyze data.

Main results and the role of chance: In comparisons between all mice (i.e., males and females $=0.03)$, discrimination $(\mathrm{p}=0.001)$, and memory $(\mathrm{p}=0.007)$. Sex-specific comparisons between the groups yielded evidence of sexual dimorphism. ICSI females exhibited a deficit in acquisition learning relative to CTL females $(\mathrm{p}<0.001)$ but there was not a significant difference between CTL and ICSI males. In the discrimination and memory tasks, ICSI males exhibited deficits relative to CTL males $(\mathrm{p}=0.002$ and $\mathrm{p}=0.02$, respectively) but the differences between females in these tasks were not significant. There was no difference in discrimination or memory retention/re-acquisition assessments conducted with mice at 12 months of age. ICSI males and females weighed significantly more than CTL counterparts at all points during the experiment.

Limitations, reasons for caution: The study was not blinded. All learning assessments utilized fully characterize learning in ICSI mice and speculate regarding the implications for cognitive function in humans conceived via ICSI.

Wider implications of the findings: Studying learning and memory processes in mouse models

61 has the potential to shed light on ICSI outcomes at the level of cognitive function. Future research should use multiple learning paradigms, assess both males and females, and investigate the effects of variables related to the ICSI procedure. Studying cognitive function in ICSI is an interdisciplinary endeavor and requires coordination between researchers at the genetic and 
66 Study funding/competing interest(s): This work was supported, in part, by grants from NIH

67 (P30GM110767, HD071736 and HD085506 to WY), the Templeton Foundation (Grant ID:

6861174 to WY), and a New Scholarly Endeavor Grant from the University of Nevada, Reno

69 Office of Research and Innovation (to ML, YW, HZ, LH, and WY). The authors declare no

70 competing interests.

71

72 Keywords: intracytoplasmic sperm injection, assisted reproductive technology, operant learning,

73 associative learning, behavior, mouse models, interdisciplinary research 


\section{Introduction}

Intracytoplasmic sperm injection (ICSI) is an assisted reproductive technology (ART) that is achieved through the injection of a single spermatozoon directly into the cytoplasm of an oocyte. ICSI has proven to be effective in treating severe forms of male factor infertility that are difficult to treat with other ARTs. Since the first ICSI pregnancies in 1992 (Palermo et al., 1992), the procedure has grown in popularity and is now the most commonly used ART worldwide (Rozenwaks \& Pereira, 2017). In the United States, ICSI use increased from $36.4 \%$ of all fertility treatment cycles in 1996 to $76.2 \%$ in 2012 (Boulet et al., 2015). Although ICSI was originally developed specifically to treat infertility related to semen quality, the use of ICSI for non-male factor infertility has also increased from $15.4 \%$ in 1996 to $66.9 \%$ in 2012 (Boulet et al., 2015). The use of ICSI as the treatment of choice for various types of infertility has raised concerns regarding its overuse, especially in light of the possibility of adverse postnatal outcomes (Esteves et al., 2018). ICSI has been responsible for over two million births since its inception (Palermo et al., 2017). As the earliest ICSI babies are now reaching maturity, researchers have become increasingly concerned with examining ICSI outcomes in various domains. Human outcome studies inherently contain many confounds and biases and therefore must be interpreted with caution (Fauser et al., 2014; Pereira et al., 2017). Nevertheless, some studies have found that ICSI may be associated with increased risks of chromosomal and epigenetic irregularities (Manipalviratn et al., 2009; Odom \& Segars, 2010), congenital birth defects (Lacamara et al, 2017; Massaro et al., 2015; Pandey et al., 2012), and cognitive disorders (Hansen et al., 2018; Sandin et al., 2013).

While some studies have suggested a tentative relationship between ICSI and abnormal psychological development, it is particularly difficult to draw conclusions regarding this 
relationship from human outcome studies because cognitive development is profoundly influenced by individuals' environmental circumstances (Hart \& Risley, 1995; Novak \& Peláez, 2004). The heterogeneity of the cultural, familial, and educational environments of children conceived via ICSI makes it impossible to extricate the respective contributions of genetic/epigenetic and environmental variables on psychological development. Characterizing the relationship between ICSI and psychological function would ideally involve studying learning and cognitive development in individuals conceived via ICSI in well-controlled environments.

This approach is not feasible with humans, but animal models provide an opportunity to control for many environmental factors and study behavior and learning processes that serve as a common basis for cognitive function in humans and nonhumans alike. We were able to identify only two studies that compared ICSI mice to naturally-conceived control (CTL) mice for this purpose. Fernández-Gonzalez et al. (2008) compared ICSI and CTL CD-1 male and female mice in a series of behavioral assays that included an open field test to assess locomotion, an elevated plus maze task to assess sensitivity to anxiety-inducing stimuli, and a free-choice y-maze task to assess habituation to novelty. They found no differences between ICSI and CTL males in any of the procedures, but ICSI females exhibited less exploration in the open field, increased anxiety as measured by time spent in the open arms of the elevated plus maze, and less habituation as measured by time spent in a previously explored arm of the y-maze. Kohda et al. (2011) found no significant differences between male ICSI and CTL C57BL/6 x DBA/2 (BDF1) mice in a series of tests designed to assess locomotion and sensitivity to fear- and pain-inducing stimuli. Female mice were not assessed in the latter study. 
The procedures used in the studies cited above allowed for comparisons between ICSI and CTL mice in terms of a) general activity/locomotion, b) sensitivity to anxiety- and pain-

121 inducing aversive stimuli, and c) habituation to novel environmental stimuli. All of these may 122 provide important information relevant to psychological function, but only the procedures that 123 measured habituation (Fernández-Gonzalez et al., 2008) may be considered to assess learning 124 per se. Learning is defined generally as changes in organisms' behavior with respect to particular 125 environmental events or stimuli as a result of previous experiences (Pierce \& Cheney, 2013).

126 Habituation is one of the most basic learning processes and describes situations in which an 127 animal's response to a particular environmental stimulus or event decreases with repeated 128 exposure to that stimulus or event (Groves \& Thompson, 1970; Rankin et al., 2009; Thompson \& 129 Spencer, 1966). Habituation is categorized as an example of nonassociative learning because 130 changes in behavior occur simply through exposure to an environmental stimulus (Domjan, $1312015)$.

133 all organisms, including humans (Domjan, 2015; Ginsburg \& Jablonka, 2010; Mackintosh, 134 1974). There are two fundamental associative learning processes that have been studied 135 extensively with both humans and nonhumans since the early 1900s: Pavlovian learning 136 (Domjan, 2005; Pavlov, 1927/1960; Rescorla, 1988) and operant learning (Pierce \& Cheney, 137 2013; Skinner, 1938, 1953; Thorndike, 1911). In Pavlovian learning, organisms learn about 138 relations between environmental stimuli. If two stimuli frequently occur together in organisms' 139 environments, they come to respond to the two stimuli in a similar fashion. This allows 140 organisms to prepare for and more effectively interact with biologically important stimuli 141 (Domjan, 2005). In operant learning, organisms learn about relations between their behavior and 
142 its effects on the environment. Responses that regularly produce rewarding consequences (e.g.,

143 the opportunity to eat food, drink water, or escape from aversive stimuli) will come to occur

144 more frequently in the environmental settings where they have been associated with these

145 consequences. Responses that do not produce rewarding consequences, or result in exposure to

146 aversive events, come to occur less frequently. Pavlovian and operant learning allow organisms

147 to interact with their environments effectively and adapt to changes in the environment that occur

148 during their lifetimes. These learning processes serve as the basis for language and other forms

149 of complex human behavior (De Houwer et al., 2016; Jablonka \& Lamb, 2014; Sturdy \&

150 Nicoladis, 2017).

To date, there have been no studies that compared associative learning between ICSI and

154 outcome studies. The purpose of the present study was to conduct the first assessment of operant

155 learning and memory in a mouse model of ICSI. ICSI and naturally-conceived CTL mice were

156 exposed to a series of operant learning procedures that assessed acquisition of a new behavior,

157 discrimination learning, and memory. These assessments were conducted while the mice were

158 between 3-6 months of age. Follow-up assessments were then conducted with some of the mice

159 to investigate retention and re-acquisition of learned performances when the mice were 12

160 months of age. 
All animal work was performed following the protocol approved by the Institutional of age) CD-1 mice used in this study were purchased from Charles River, and housed under pathogen-free conditions in a temperature- and humidity- controlled animal facility at the University of Nevada, Reno. Natural mating was set up by placing one adult male into a cage with one adult female, and all of the naturally-conceived control (CTL) mice used in this study were those from the first 4 litters of four breeding pairs. Pups were weaned at 3 weeks after birth.

\section{Intracytoplasmic Sperm Injection (ICSI) Mice}

Adult female CD-1 mice at 6-12 weeks of age with body weight ranging between 25-45 grams were used as either egg donors or recipients/surrogates. These female mice were superovulated by intraperitoneal injection of 7 IU of Pregnant Mare's Serum Gonadotropin (PMSG), followed by intraperitoneal injection of 7 IU of human Chorionic Gonadotropin (hCG) 2 min. The cumulus-free oocytes were washed and kept in the KSOM+AA medium (Millipore, before ICSI. 
187

188

189

190

191

192

193

194

195

196

197

198

199

200

201

202

203

204

205

206

207

208

209

medium. The top $100 \mu \mathrm{l}$ sperm suspension was sonicated at the medium level for five times with 3 seconds each (Bioruptor UCD-200; Diagenode). An aliquot of $2 \mu 1$ sperm HTF suspension was mixed immediately with $50 \mu \mathrm{l}$ of 4\% PVP (Sigma, Cat\# P5288) in water (Millipore, Cat\# TMS006-C). A single sperm head was picked up and injected into the mature oocytes using a glass pipette equipped with a piezo drill under the control of an electric micromanipulator (TransferMan NK2, Eppendorf). Injection of $\sim 20$ oocytes was completed within 20 minutes at room temperature. Sperm sonication was then repeated to obtain freshly prepared sperm heads for injection. Injected oocytes were transferred to the KSOM+AA medium (Millipore, Cat\# MR-121-D) covered by mineral oil and cultured in an incubator at $37^{\circ} \mathrm{C}$ with humidified air containing 5\% $\mathrm{CO}_{2}$. Between 4-6 h post ICSI, 18-26 2PN stage embryos were transferred into the oviducts of pseudo-pregnant CD-1 females (8-16 weeks of age) that had been mated during the prior night with vasectomized adult CD-1 males (10-16 weeks of age).

\section{Subjects}

36 ICSI (18 males and 18 females) and 37 naturally-conceived CTL mice (19 males and 18 females) obtained as described above served as the subjects. All the mice were between 12-13 weeks of age at the beginning of the training described below.

\section{Housing}

ICSI and CTL mice were housed separately in clear plastic Tecniplast ${ }^{\circledR}$ home cages in same-sex groups of three to five mice per cage. Cages were equipped with absorbent corn cob bedding and items for enrichment including cotton fiber nestlets, a transparent red polycarbonate mouse hut and wooden gnawing sticks. Cages were housed in a temperature- and humidity- 
210

211

212

213

214

215

216

217

218

219

220

221

222

223

224

225

226

227

228

229

230

231

232

controlled colony room with a 12:12 light/dark cycle with lights on at 7:00. Except for the scheduled deprivations, subjects had free access to laboratory chow (Harlan Teklad) in overhead feeders. Subjects had free access to purified drinking water at all times.

\section{Food Deprivation}

In order to establish motivation for the sucrose pellet rewards used in experimental sessions, subjects were deprived of food $14 \mathrm{~h}$ prior to daily experimental sessions. Food was removed from the subjects' cages daily at 19:00. Mice had free access to water during the food deprivation period. Experimental sessions were conducted daily at 9:00, and food was returned to the cages after all mice had completed their training sessions. They then had free access to food and water until the next deprivation period.

\section{Handling and Weighing}

Mice were handled using $15 \mathrm{~cm}$ tall x $5.75 \mathrm{~cm}$ diameter clear plastic tubes open on one end and wide enough to allow the subjects to move freely while sitting in the bottom. Handling tubes have been shown to reduce inter-handler variability and handler-induced stress (Hurst \& West, 2010). Prior to each session, a mouse was guided into the tube, weighed, and then placed in the experimental apparatus. When the session concluded, the mouse was transported back to its home cage in the tube.

\section{Apparatus}

All learning and memory assessments were conducted in Med Associates ${ }^{\circledR}$ (St. Albans, VT) modular operant test chambers (ENV-307A). The inside dimensions of the chambers were 
$12.7 \mathrm{~cm}$ high x $15.9 \mathrm{~cm}$ wide $\mathrm{x} 14.0 \mathrm{~cm}$ deep. Side walls were composed of transparent polycarbonate, and the front and back walls were composed of three modular columns of aluminum panels. Each chamber was housed in a sound attenuating cabinet with a ventilation fan to mask ambient noise. A $100 \mathrm{~mA}$ house light (ENV-315M) was mounted in the center column of the back wall of the chambers $10 \mathrm{~cm}$ above the grid floor. On the front wall of the chambers, opposite of the house light, a receptacle measuring $3.8 \mathrm{~cm}$ high x $8.9 \mathrm{~cm}$ wide was mounted in the center column $0.5 \mathrm{~cm}$ above the grid floor. The receptacle was capable of receiving $20 \mathrm{mg}$ Bio-Serv sucrose reward pellets delivered via a pedestal mount pellet dispenser (ENV-203M-20). Two illuminable nose poke operanda (ENV-313M) were mounted $3 \mathrm{~cm}$ to either side of the receptacle. The access port for each nose poke measured $1.3 \mathrm{~cm}$ in diameter $\mathrm{x} 1 \mathrm{~cm}$ deep. Entry of a subjects' nose at least $0.64 \mathrm{~cm}$ into the access port broke a photobeam and defined a response. The presentation and recording of all experimental events were controlled via MED-

245 PC IV (Med Associates) software.

Prior to the learning and memory assessments described below, magazine training was provided to teach the subjects to approach the food receptacle and eat when reward pellets were

250 delivered. Subjects were 12-13 weeks of age at the onset of this training and were deprived of

251 food prior to all sessions as described above. Once an animal was placed inside the chamber, a

252 single pellet was delivered when the animal was oriented toward the receptacle but did not have 253 its head inside of it. After the animal approached and ate the pellet, another pellet was delivered 254 in the same manner. A session was terminated when a mouse had consumed seven pellets. The 
mouse received two such sessions per day for five consecutive days (10 total sessions). By the end of this training, all subjects reliably approached the receptacle and consumed pellets when they were delivered.

\section{Learning and Memory Assessments}

Subjects were exposed to four operant learning and memory assessments conducted in succession. These procedures were the same as those described in Lewon et al. (2017). Each successive assessment was designed to evaluate an increasingly complex performance. These are described below.

\section{Nose Poke Acquisition}

The first assessment was designed to evaluate the acquisition of a new response through reinforcement. Reinforcement describes a fundamental learning process whereby the frequency of a behavior increases because it has been followed by a rewarding consequence (Domjan, 2015). In the present study, the behavior to be acquired was nose poking (i.e., insertion of the nose at least $0.64 \mathrm{~cm}$ into the portal of the nose poke operanda) and the rewarding consequence was the delivery of a sugar pellet. The frequency with which this behavior increased through reinforcement and occurred across training sessions provided a measure of acquisition learning. Subjects were 12.5-13.5 weeks of age at the beginning of this assessment. Each session began with the illumination of the house light and both nose poke stimulus lights. Responses on either nose poke were immediately followed by the delivery of one sucrose pellet (i.e., a fixedratio 1 schedule of reinforcement). Each session was terminated after 15 minutes. One session was conducted daily across 10 consecutive days. 


\section{Switching Discrimination Task}

The purpose of the second procedure was to assess discrimination learning.

Discrimination occurs when organisms learn to engage in a response when the probability of 
nose poke, regardless of position (thus the name switching discrimination task; SDT). Sessions were terminated after 15 minutes, and one session was conducted daily for 20 consecutive days.

$$
\text { Discrimination index (DI) provided a measure of the extent to which this discrimination }
$$

performance was learned. DI was calculated by dividing the total number of responses on the

\section{Delayed Non-Matching-To-Position Memory Task}

317 On the other hand, reference memory refers to the longer-term retention of information that

318 allows for the successful use of shorter-term working memory in the completion of a task. appropriately use working memory (Domjan, 2015).

322 of the house light and the start of a trial in which one of the two nose pokes was illuminated $(0.5$ 
required to respond on the illuminated nose poke to proceed to the subsequent portions of the trial. If they responded on the unilluminated nose poke, there were no programmed consequences. A response on the illuminated nose poke initiated a 2-s retention interval during which both nose pokes were dark Any responses that occurred during this interval produced no programmed consequences. Following the retention interval, both nose pokes were illuminated for the free choice portion of the trial, and subjects could respond on either nose poke. Responses on the same nose poke as required during the forced choice portion of the trial were counted as incorrect and no reward was delivered. Responses on the opposite nose poke of the forced choice trial were counted as correct and rewarded with the delivery of a sugar pellet (thus the name nonmatching-to-position). A trial ended after a correct or incorrect response on the free choice portion and was followed a 5-s ITI. After the ITI, the next trial began with another forced choice. Sessions were terminated when an animal completed 20 trials or 30 minutes, whichever occurred first. Subjects were 17-18 weeks of age at the beginning of this training and received one session daily for 30 consecutive days.

In order to obtain rewards in a trial, mice were required to respond on the nose poke that was not the one on which they responded in the forced choice portion. The working memory aspect of this performance was that the mice had to remember where they had responded in the

341 forced choice portion of the trial during the retention interval. The reference memory portion

342 involved remembering the general rule for reward: respond on the nose poke opposite of the one 343 on which they responded during the forced choice portion of the trial, whether it occurred on the 344 left or right nose poke. When the mice did so, they received a sugar pellet reward and the trial 345 was counted as "correct." The proportion of correct trials per session provided a measure of memory performance. 


\section{DNMTP Retention Checks}

After the 30 trials of DNMTP training described above, mice were removed from the training environment for a prescribed period of time before receiving three additional DNMTP retention check sessions to assess long-term memory of the DNMTP performance. Sessions were identical to those described above. The first retention check occurred two days after the last checks.

\section{Follow-Up Assessments with Aged Mice}

After the initial battery of assessments, follow-up assessments were conducted with some of the same mice from the initial assessments (CTL $n=17 ; 10$ males, 7 females; ICSI $n=16,9$ males, 7 females) when they were between 52-53 weeks of age (i.e., approximately 30 weeks

361 after the last DNMTP retention check session). Prior to the follow-up assessments, mice were weighed for five days under free-feeding conditions starting at 52 weeks of age. After five days, the food deprivation schedule described above was imposed and assessments commenced. The

364 follow-up assessments consisted of 15 daily sessions of the switching discrimination task

365 followed immediately by 15 daily sessions of the DNMTP memory task. All subjects had

366 previous exposure to these procedures during their initial training, and the follow-up assessments

367 were therefore designed to test retention and re-acquisition of these performances at old age.

\section{Statistical Analysis}


Mixed repeated measures ANOVAs were used to compare the results for ICSI and CTL

371 mice in each learning and memory assessment. The between-subjects factor in these analyses

372 was group (ICSI vs. CTL) and the within-subjects factor was session. Omnibus analyses were used to compare all ICSI and CTL mice, and these were followed by sex-specific analyses (i.e., ICSI vs. CTL males and ICSI vs. CTL females). The analyses tested for main effects of group and session as well as for a group $\mathrm{x}$ session interaction. We used an $\alpha$ value of 0.05 as the criterion for significance, and partial-eta squared values $\left(\eta p^{2}\right)$ are provided as estimates of effect 377 sizes.

\section{Results}

\section{Nose Poke Acquisition}

The training sessions in this phase of the experiment were designed to assess the

ICSI and CTL males, but CTL females consistently made more responses per session than their

ICSI counterparts. This means that the slight overall difference between all ICSI and CTL mice number of responses per session between ICSI and CTL females during this procedure.

392 females combined) found a large effect for session $\left(F_{9,639}=44.31, p<0.001, \eta p^{2}=0.38\right)$ and 
393

394

395

396

397

398

399

400

401

402

403

404

405

406

407

408

409

410

411

412

413

414

415

smaller effects for group $\left(\mathrm{F}_{1,71}=4.93, \mathrm{p}=0.03, \eta \mathrm{p}^{2}=0.07\right)$ and the group $\mathrm{x}$ session interaction

$\left(\mathrm{F}_{9,639}=2.06, \mathrm{p}=0.03, \eta \mathrm{p}^{2}=0.03\right)$. The same analysis was used to compare ICSI and CTL

males and found a large effect for session $\left(\mathrm{F}_{9,315}=20.54, \mathrm{p}<0.001, \eta \mathrm{p}^{2}=0.37\right)$. There was a

barely significant effect for the group $\mathrm{x}$ session interaction $\left(\mathrm{F}_{9,315}=1.96, \mathrm{p}=0.05, \eta \mathrm{p}^{2}=0.05\right)$,

but there was no main effect for group. The comparison between ICSI and CTL females found significant main effects for session $\left(\mathrm{F}_{9,306}=28.46, \mathrm{p}<0.001, \eta \mathrm{p}^{2}=0.46\right)$ and group $\left(\mathrm{F}_{1,34}=\right.$

$\left.6.98, \mathrm{p}=0.01, \eta \mathrm{p}^{2}=0.17\right)$ but no significant group $\mathrm{x}$ session interaction.

To summarize, there was little difference in acquisition between ICSI and CTL males, but the CTL females acquired nose poke responding more readily than the ICSI females. While the CTL females consistently made more responses per session than ICSI females, the statistical analysis did not find a significant group x session interaction. It appeared that CTL females consistently responded more than ICSI females, but the degree to which responding increased across sessions was similar for both groups of females.

\section{Switching Discrimination Task}

The switching discrimination task (SDT) assessed discrimination learning. Figure 2 shows the mean discrimination index (DI) for all ICSI and CTL mice (top) and for ICSI/CTL males and females (bottom) in the SDT procedure. DI increased for all mice across the 20 training sessions. While both groups gradually made fewer unrewarded responses during this training, the top panel shows that the CTL mice made a greater proportion of rewarded responses from the third session onward and reached a substantially higher DI by the final session $(0.68$, +/- 0.02 SEM for CTL compared to $0.58,+/-0.02$ SEM for ICSI). The graphs in the bottom panels of Figure 2 show that both male and female CTL mice often had higher DIs than their 
416

417

418

419

420

421

422

423

424

425

426

427

428

429

430

431

432

433

434

435

436

437

438

ICSI counterparts, but the difference between CTL and ICSI discrimination performances was more pronounced and consistent for males.

Statistical analysis for the comparison between all ICSI and CTL mice found a large main effect for session $\left(F_{19,1349}=100.63, \mathrm{p}<0.001, \eta p^{2}=0.59\right)$ and a main effect for group $\left(F_{1,71}=\right.$ $\left.11.77, \mathrm{p}=0.001, \eta \mathrm{p}^{2}=0.14\right)$, but no effect for the group $\mathrm{x}$ session interaction. Similarly, the comparison between ICSI and CTL males found significant main effects for session $\left(\mathrm{F}_{19,665}=\right.$ $\left.71.16, \mathrm{p}<0.001, \eta \mathrm{p}^{2}=0.67\right)$ and group $\left(\mathrm{F}_{1,35}=11.10, \mathrm{p}=0.02, \eta \mathrm{p}^{2}=0.24\right)$ but no group $\mathrm{x}$ session interaction. The comparison between ICSI and CTL females found a significant main effect for session $\left(\mathrm{F}_{19,646}=35.96, \mathrm{p}<0.001, \eta \mathrm{p}^{2}=0.51\right)$ but no main effect for group or the group x session interaction.

Taken together, CTL mice exhibited better discrimination learning, and this difference was more pronounced between CTL and ICSI males than it was between the female groups.

Despite this, statistical analyses did not reveal significant group x session interactions for any of the comparisons, including the comparison between CTL and ICSI males. Overall, it appeared that the rate of improvement in DI scores across sessions was similar for the two groups, but the CTL mice nevertheless had consistently higher DI scores.

\section{Delayed Non-Matching-to-Position Memory Task}

This procedure assessed working and reference memory. Figure 3 shows the mean proportion of correct/rewarded trials in DNMTP recognition memory sessions for all ICSI and CTL (top panel) and for ICSI/CTL males and females (bottom panels). The top panel shows that while the proportion of correct responses made by both groups increased across sessions, the

CTL mice consistently made more correct responses from the seventh session onward. As in the 
439

440

441

442

443

444

445

446

447

448

449

450

451

452

453

454

455

456

457

458

459

460

previous SDT procedure, there appeared to be a larger difference in performance between males than females. CTL males made a greater proportion of correct responses than ICSI males in every session except the second. On the other hand, CTL and ICSI females made approximately the same proportion of correct responses until the $11^{\text {th }}$ session, after which CTL females made slightly more correct responses in most sessions.

The mixed repeated measures ANOVA comparing all ICSI to CTL found significant main effects for session $\left(F_{29,2030}=19.72, p<0.001, \eta p^{2}=0.22\right)$ and group $\left(F_{1,71}=7.67, p=\right.$ $0.007, \eta p^{2}=0.10$ ) but not for the group x session interaction. The comparison between CTL and ICSI males similarly found significant effects for session $\left(F_{29,986}=10.80, p<0.001, \eta p^{2}=0.24\right)$ and group $\left(F_{1,71}=6.44, p=0.02, \eta p^{2}=0.16\right)$ but not for the group $x$ session interaction. For the comparison between CTL and ICSI females, there was an effect for session $\left(\mathrm{F}_{29,986}=9.59, \mathrm{p}<\right.$ $\left.0.001, \eta p^{2}=0.22\right)$ but not for group or the group $\mathrm{x}$ session interaction.

The results of the DNMTP memory procedure were similar to those obtained in the preceding SDT. Specifically, CTL mice performed better than ICSI, and the difference between CTL and ICSI males was more pronounced than the difference between CTL and ICSI females. Statistical tests again found significant main effects for the group factor in the comparison between all ICSI/CTL and between male ICSI/CTL, but there was not a significant group $\mathrm{x}$ session interaction. Thus, it appeared that ICSI and CTL performance improved at approximately the same rate across training sessions, but the CTL mice (especially the males) consistently made a greater proportion of correct responses than their ICSI counterparts.

\section{DNMTP Retention Checks}


Retention of the DNMTP performance was assessed with three retention check sessions.

462

463

464

465

466

467

468

469

470

471

472

473

474

475

476

477

478

479

480

481

482

483

Figure 4 shows the mean proportion of correct responses in the three DNMTP retention checks

for all ICSI and CTL mice (top) and separated by males and females (bottom). For reference, the first (leftmost) data point on these figures represents the mean proportion correct for each group in the last five DNMTP training sessions (i.e., sessions 25-30). The top panel shows that the mean proportion correct decreased slightly for both groups in the first (2-day) retention check relative to the last five sessions of DNMTP training. For CTL mice, the mean proportion correct continued to decrease slightly across the remaining two retention checks while the ICSI mice' performance remained at approximately the same level. The two groups' performances were equal in the final 10-day retention check. The bottom panels of Figure 4 shows that the proportion correct decreased monotonically for CTL males and females across the retention checks. For ICSI males, the proportion correct in the 5-day test increased slightly relative to the 2-day test but decreased to approximately the same level as the CTL males in the 10-day test. For ICSI females, proportion decreased across the 2- and 5-day tests but increased slightly in the final test.

The mixed ANOVA comparing all ICSI and CTL mice found a significant effect for session (i.e., significant decreases in proportion correct across the three retention checks; $F_{2,140}=$ $\left.6.17, p=0.003, \eta p^{2}=0.08\right)$ but no effects for group or group $x$ session interaction. The comparisons between ICSI and CTL males and females likewise found significant effects for session for both $\left(F_{2,68}=3.17, p=0.05, \eta p^{2}=0.09\right.$ for males and $F_{2,68}=3.65, p=0.03, \eta p^{2}=$ 0.10 for females), but found no effects for group or group x session interaction for either. Thus, while there was a general decrease in proportion correct across the three retention checks, there was no significant difference between the groups in the rate at which this decrease occurred. 


\section{Follow-Up Assessments with Aged Mice}

Follow-up assessments were conducted with aged mice to evaluate long-term retention

The right panel of Figure 6 shows performance in the DNMTP memory reassessments. session, group, or group x session interaction.

\section{Body Weight}

As noted above, mice were weighed immediately prior to all sessions following a 14-h 
experiment, ICSI males and females both consistently weighed more than their CTL

508

509

510

511

512

513

514

515

516

517

518

519

520

521

522

523

524

525

526

527

528 counterparts. Both ICSI and CTL males gained weight across the experiment, but ICSI males gained weight at a greater rate than CTL males. Compared to the males, the females gained relatively little weight across the experiment. However, both ICSI and CTL females gained a larger proportion of weight during the retention checks when they had longer periods of access to food. From the last DNMTP session to the final retention check, the weights for ICSI and CTL males increased by $3.94 \%$ and $3.42 \%$, respectively. In comparison, ICSI female weights increased by $10.79 \%$ and CTL female weights increased by $7.88 \%$ during the same period.

Figure 7 displays the mean weights of the mice for five days prior to and during the reassessment training sessions starting at 52 weeks of age. All mice had ad libitum access to food from the end of the learning and memory initial assessments (when they were approximately six months of age) to the time of the re-training, when the food deprivation regimen was reinstated. At the first weighing after six months of free-feeding, ICSI males weighed an average of $62.4 \mathrm{~g}$ (+/- 3.10 SEM) compared to 50.6 g (+/- 2.96 SEM) for CTL males. ICSI females likewise weighed substantially more than their female CTL counterparts (63.7 $\mathrm{g}+/-6.25$ SEM for ICSI compared to $46.3 \mathrm{~g}+/-4.67$ for CTL).

The reinstatement of the food deprivation schedule produced an immediate reduction in weights of the males, but weights stayed largely the same until the end of the reassessments 30 days later. For females, the food deprivation schedule resulted in progressively lower weights across this same time, and this was more pronounced for the CTL females. 
We subjected ICSI and CTL mice to a series of operant learning procedures to assess acquisition, discrimination learning, and memory. The inclusion of both males and females

531 allowed for global comparisons between ICSI and CTL mice as well as for same-sex comparisons between the groups. Overall, CTL mice were found to outperform their ICSI counterparts in all but one of the learning and memory tasks we employed during their initial

534 training, and the differences were largely due to sex-specific differences in performance in the tasks. Specifically, CTL females performed better during acquisition learning than ICSI females,

536 but there was no difference in acquisition between ICSI and CTL males. In the SDT and

537 DNMTP procedures, CTL males exhibited superior discrimination learning and memory

538 compared to their ICSI counterparts, but there was not a statistically significant difference

539 between ICSI and CTL females in these tasks. There were no apparent differences between the 540 groups in the DNMTP retention checks designed to assess longer-term memory. Both groups

541 showed significant decrements in performance in SDT and DNMTP re-training sessions 542 conducted at 52 weeks of age.

While CTL mice exhibited superior performance in all procedures except the DNMTP 544 retention checks during initial training, it is interesting to note that statistical analyses revealed 545 significant group effects but no significant effects for group x session interactions. This means 546 that the extent to which performance increased across training sessions was roughly equivalent 547 for ICSI and CTL in the procedures employed here. Despite similar changes in behavior across 548 repeated exposures to the learning and memory assessments, CTL mice consistently performed 549 at a higher level. At this point it is unclear why this was the case. Further research investigating 550 basic learning processes with these mice will be required to explain this difference. 
A notable auxiliary finding was the relatively large and consistent difference in weights between ICSI and CTL mice. ICSI males and females both weighed more than their CTL counterparts both during initial training when mice were three to six months of age and when mice were over a year old. Other studies have similarly reported higher weights at birth for ICSI B6C3F1 males and females relative to CTL (Scott et al., 2010) as well as significantly higher

556 weights for ICSI CD-1 females relative to CTL females from approximately 15 weeks of age

557 (Fernández-Gonzalez et al., 2008). These data suggest that further investigations into potential

558 metabolic differences between ICSI and CTL mice may be warranted.

564 would add an additional level of rigor and control for any inadvertent differences in how mice

were handled. A second limitation is that the procedures were conducted in succession, meaning that each individual assessment occurred when the mice were at a single age. It may be the case

567 that comparing acquisition, discrimination, or memory between ICSI and CTL mice at different 568 points in the developmental timeline may yield different results. As a proof of concept study, we 569 aimed to show the potential effects of the overall ICSI procedure on the health of offspring; thus, 570 we did not distinguish multiple factors involved in ICSI, e.g., superovulation protocol, sperm 571 preparation protocol, culture conditions, injection conditions, stages for embryo transfer, and the 572 age of surrogate mothers. These variables may be worth testing in future studies. 

memory in animal models has the potential to shed light on outcomes of ICSI at the level of

575 cognitive function. Our data open up a number of avenues for further investigation. In this study, we investigated operant learning and memory using only reinforcement procedures in which sugar pellets served as the reward. Studies have shown that mouse models that exhibit

578 learning deficits relative to control mice in one type of operant procedure may exhibit superior 579 performance in a different operant learning paradigm (Lewon et al., 2017). It is therefore 580 necessary to expose mouse models to as many types of learning situations as possible to obtain 581 the fullest picture of cognitive function. Operant learning assessments are diverse and include 582 procedures that use other types of rewards under different schedules of reinforcement, different types of spatial and multisensory discrimination and memory tasks, escape/avoidance learning tasks, and procedures that provide measures of sensitivity to stress-inducing aversive events. In

585 addition to operant learning procedures, future studies may also examine more basic processes 586 such as nonassociative and Pavlovian learning. One benefit of the modular experimental 587 chambers such as those used in this experiment is that a single apparatus may be readily modified to accommodate all of these types of assessments. As there appeared to be sex-specific 589 differences in learning and memory in this experiment and studies have similarly found evidence 590 of sexual dimorphism in other measures of ICSI outcomes (Esteves et al, 2018; Fernández591 Gonzalez et al., 2008), this research should include assessments of both males and females 592 (Shansky, 2019). In addition to studying ICSI mice with other types of learning procedures, future research 594 may also examine how variables related to the ICSI procedure itself may affect learning and memory. Some studies have found that ART is associated with an increased occurrence of 
epimutations and imprinting disorders (de Waal, et al., 2012; Lazaraviciute et al., 2014; Pinborg, 2016), and it is known that ARTs may induce embryonic stress responses that alter gene expression and exert a number of other epigenetic effects during early development (Ramoscontribute to the likelihood of epigenetic alterations (Esteves et al., 2018; Ghosh et al., 2017; to produce modifications in gene expression that affect neurodevelopment and psychological function across the lifespan (Grigorenko et al., 2016; Guan et al., 2015), and there is evidence that some of these modifications may be inherited by offspring (Babenko et al., 2015; Chen et al., 2016; Nestler, 2016; Jablonka \& Raz, 2009). For all of these reasons, future research should investigate how the ICSI procedure and the epigenetic factors associated with it affect cognitive

608 function, ideally across multiple generations. and the psychological development of ICSI humans. Although ICSI mice exhibited certain learning and memory deficits relative to CTL mice in the testing we employed, cognitive deficits

612 should not be assumed to be invariably associated with ICSI in humans. There are several

613 reasons for this. First, as noted above, the assessments conducted here represent a small portion

614 of the procedures available for investigating learning and memory, and a wider range of these

615 will be needed to more fully characterize cognitive function in ICSI mice. Second, human

616 learning environments differ in important ways from mice (Hayes \& Delgado, 2007), and

617 families of ICSI children vary widely in terms of socioeconomic status, education, and access to 
medical and educational resources for their children. The deficits observed in ICSI mice in this study may therefore prove to be clinically insignificant in certain social environments.

Finally, and perhaps most importantly, cognitive function must be seen as the product of a complex set of interactions between individuals and their environments throughout the lifespan. During development, environmental factors interact with genetic materials to determine the physiological phenotypes of whole individuals. These individuals then interact with their physical and social environments, which shape their behavior across time through nonassociative, Pavlovian, and operant learning processes. Different learning environments will inevitably impart different repertoires, and the physiological characteristics of individuals (e.g., brain function, metabolism, sensory abilities, etc.) determine their capacity for learning from particular types of environmental contingencies. Physiological characteristics that provide advantages for learning in certain environments may prove to be detrimental in others (Lewon et al., 2017). For these reasons, studying the relationship between ICSI and cognitive function is a truly interdisciplinary endeavor that does not fall solely within the domain of either genetics or psychology (Hayes \& Fryling, 2009). Genetic and epigenetic analyses by themselves cannot explain cognitive development in a directly causal manner, as this depends in large part upon the types of interactions individuals have with their environments. Similarly, analyses at the psychological level alone cannot explain differences in learning capacities related to genetic characteristics. Further interdisciplinary research on basic learning processes with mouse models has the potential to enhance our understanding of these interactions as they relate to ICSI and other ARTs. This research will require close coordination between investigators at both the genetic and psychological levels of analysis. 
643 Cohen, Kristen Green, Osmar Lopez, Elisabeth Mclean, Haley Mizell, Caitlyn Peal, Keenan

644 Raquel, Tori Sandoval, Emily Spurlock, Melanie Stites, and Jamiika Thomas for technical 645 assistance.

646

647

648 649 Learning and memory assessments and data analysis were conducted by ML, CP, and MP.

\section{Role of Authors}

ML, YW, CP, and MP contributed to the initial draft of the manuscript, and subsequent edits were made by all authors. ML, YW, CP, HZ, LH, and WY contributed to the conception and design of the study. The ICSI procedure and breeding were conducted by YW and HZ. 
652

653

654

655

656

657

658

659

660

661

662

663

664

665

666

667

668

669

670

671

672

673

674

\section{References}

Babenko O, Kovalchuk I, Metz GAS. Stress-induced perinatal and transgenerational epigenetic programming of brain development and mental health. Neuroscience and Biobehavioral Reviews 2015;48:70-91.

Boulet SL, Mehta A, Kissin DM, Warner L, Kawwass JF, Jamieson DJ. Trends in use of and reproductive outcomes associated with intracytoplasmic sperm injection. Journal of the American Medical Association 2015;313:255-263.

Chen Q, Yan W, Duan E. Epigenetic inheritance of acquired traits through sperm RNAs and sperm RNA modifications. Nature Reviews: Genetics 2016;17:733-743.

De Houwer J, Hughes S, Barnes-Holmes D. Associative learning as higher order cognition:

Learning in human and nonhuman animals from the perspective of propositional theories and relational frame theory. Journal of Comparative Psychology 2016;130:215-225.

de Waal E, Yamazaki Y, Ingale P, Bartolomei MS, Yanagimachi R, McCarrey JR. Gonadotropin stimulation contributes to an increased incidence of epimutations in ICSI-derived mice. Human Molecular Genetics 2012;21:4460-4472.

Domjan M. Pavlovian conditioning: A functional perspective. Annual Review of Psychology 2005;56:179-206.

Domjan M. The Principles of Learning and Behavior. $7^{\text {th }}$ edn, 2015. Cengage, Stamford, CT.

Esteves SC, Roque M, Bedoschi G, Haahr T, Humaidin P. Intracytoplasmic sperm injection for male infertility and consequences for offspring. Nature Reviews: Urology 2018;15:535562.

Fauser BCJM, Devroey P, Diedrich K, Balaban B, Bonduelle M, Delemarre-van de Waal HA, Estella C, Ezcurra D, Geraedts JPM, Howles CM et al. Health outcomes of children born 
675

676

677

678

679

680

681

682

683

684

685

686

687

688

689

690

691

692

693

694

695

696 after IVF/ICSI: A review of current expert opinion and literature. Reproductive

BioMedicine Online 2014;28:162-182.

Fernández-González R, Moreira PN, Pérez-Crespo M, Sánchez-Martín M, Ramirez MA, Pericuesta E, Bilbao A, Bermejo-Alvarez P, de Dios Hourcade J, de Fonseca FR et al. Long-term effects of mouse intracytoplasmic sperm injection with DNA-fragmented sperm on health and behavior of adult offspring. Biology of Reproduction 2008;78:761772.

Ghosh J, Coutifaris C, Sapienza C, Mainigi M. Global DNA methylation levels are altered by modifiable clinical manipulations in assisted reproductive technologies. Clinical Epigenetics 2017;9:1-10.

Ginsburg S, Jablonka, E. The evolution of associative learning: A factor in the Cambrian explosion. Journal of Theoretical Biology 2010;266:11-20.

Grigorenko EL, Kornilov SA, Naumova OY. Epigenetic regulation of cognition: A circumscribed review. Development and Psychopathology 2016;28:1285-1304.

Groves PM, Thompson RF. Habituation: A dual-process theory. Psychological Review 1970;77:419-450.

Guan J, Xie H, Ding X. The role of epigenetic regulation in learning and memory. Experimental Neurology 2015;268:30-36.

Hansen M, Greenop KR, Bourke J, Baynam G, Hart RJ, Leonard, H. Intellectual disability in children conceived using assisted reproductive technology. Pediatrics;142:e20181629.

Hart B, Risley TR. Meaningful differences in the everyday experience of young American children. 1995. Brookes, Baltimore, MD. 
Hayes LJ, Delgado D. Invited commentary on animal models in psychiatry: Animal models of non-conventional human behavior. Behavior Genetics 2007;37:11-17.

Hayes LJ, Fryling MJ. Interdisciplinary science in interbehavioral perspective. Behavior and Social Issues 2009;18:5-9.

Hurst JL, West RS. Taming anxiety in laboratory mice. Nature Methods 2010;7:825-828.

Jablonka E, Lamb MJ, Zeligowsi A. Evolution in four dimensions: Genetic, epigenetic, behavioral, and symbolic variation in the history of life. Revised edn. 2014. MIT Press, Cambridge, MA.

Jablonka E, Raz G. Transgenerational epigenetic inheritance: Prevalence, mechanisms, and implications for the study of heredity and evolution. The Quarterly Review of Biology 2009;84:131-176.

Kimura Y, Yanagimachi R. Intracytoplasmic sperm injection in the mouse. Biology of Reproduction 1995;52:709-720.

Kohda, T, Ogonuki N, Inoue K, Furuse T, Kaneda H, Suzuki T, Kaneko-Ishino T, Wakayama T, Wakana S, Ogura A et al. Intracytoplasmic sperm injection induces transcriptome perturbation without any transgenerational effect. Biochemical and Biophysical Research Communications 2011;410:282-288.

Lacamara C, Ortega C, Villa S, Pommer R, Schwarze JE. Are children born from singleton pregnancies conceived by ICSI at increased risk for congenital malformations when compared to children conceived naturally? A systematic review and meta-analysis. JBRA Assisted Reproduction 2017;21:251-259.

Lazaraviciute G, Kauser M, Bhattacharya S, Haggarty P, Bhattacharya S. A systematic review and meta-analysis of DNA methylation levels and imprinting disorders in children 
conceived by IVF/ICSI compared with children conceived spontaneously. Human Reproduction Update 2014;20:840-852.

Lewon M, Peters CM, Van Ry PM, Burkin DJ, Hunter KW, Hayes LJ. Evaluation of the behavioral characteristics of the $m d x$ mouse model of Duchenne muscular dystrophy through operant conditioning procedures. Behavioural Processes 2017;142:8-20.

Mackintosh NJ. The psychology of animal learning. 1974. Academic Press, London.

Manipalviratn S, DeCherney A, Segars J. Imprinting disorders and assisted reproductive technology. Fertility and Sterility 2009;91:305-315.

Massaro, PA, MacLellan, DL, Anderson PA, Romao, RL. Does intracytoplasmic sperm injection pose an increased risk of genitourinary congenital malformations in offspring compared to in vitro fertilization? Journal of Urology 2015;193:1837-1842.

Nestler EJ. Transgenerational epigenetic contributions to stress responses: Fact or fiction? PLoS Biology 2016;14:1-7.

Novak G, Peláez M. Child and adolescent development: A behavioral systems approach. 2004. Sage, Thousand Oaks, CA.

Odom LN, Segars J. Imprinting disorders and assisted reproductive technology. Current Opinion in Endocrinology, Diabetes and Obesity 2010;17:517-522.

Palermo G, Joris H, Devroey P, Van Steirteghem AC. Pregnancies after intracytoplasmic sperm injection of single spermatozoon into an oocyte. Lancet 1992;340:17-18.

Palermo GD, O’Neill CL, Chow S, Cheung S, Parrella A, Pereira N, Rosenwaks Z. Intracytoplasmic sperm injection: State of the art in humans. Reproduction, 2017;154:F93-F110. 
Pandey S, Shetty A, Hamilton M, Bhattacharya S, Maheshwari A. Obstetric and perinatal outcomes in singleton pregnancies resulting from IVF/ICSI: A systematic review and meta-analysis. Human Reproduction Update 2012;18:485-503.

Pavlov IP. Conditioned reflexes: An investigation of the physiological activity of the cerebral cortex. 1960. Dover, Mineola, New York.

Pereira N, O’Neill C, Lu V, Rosenwaks Z, Palermo GD. The safety of intracytoplasmic sperm injection and long-term outcomes. Reproduction 2017;154:F61-F70.

Pierce WD, Cheney CD. Behavior analysis and learning. $5^{\text {th }}$ edn, 2013. Psychology Press, New York, NY.

Pinborg A, Loft A, Romundstad LB, Wennerholm U, Söderström-Anttila V, Bergh C, Aittomäki K. Epigenetics and assisted reproductive technologies. Acta Obstetricia et Gynecologica Scandinavica 2016;95:10-15.

Ramos-Ibeas P, Heras S, Gómez-Redondo I, Planells B, Fernández-González R, Pericuesta E, Laguna-Barraza R, Pérez-Cerezales S, Gutiérrez-Adán A. Embryo responses to stress induced by assisted reproductive technologies. Molecular Reproduction and Development 2019;1-15.

Rankin CH, Abrams T, Barry RJ, Bhatnagar S, Clayton DF, Colombo J, Coppola G, Geyer MA, Glanzman DL, Marsland S et al. Habituation revisited: An updated and revised description of the behavioral characteristics of habituation. Neurobiology of Learning and Memory 2009;92:135-138.

Rescorla RA. Pavlovian conditioning: It's not what you think it is. American Psychologist 1988;43:151-160. 
764

765

766

767

768

769

770

771

772

773

774

775

776

777

778

779

780

781

782

783

784

785

786

Rosenwaks Z, Pereira N. The pioneering of intracytoplasmic sperm injection: Historical perspectives. Reproduction 2017;154:F71-F77.

Sandin S, Nygren K, Iliadou A, Hultman CM, Reichenberg A. Autism and mental retardation among offspring born after in vitro fertilization. Journal of the American Medical Association 2013;310:75-84.

Scott KA, Yamazaki Y, Yamamoto M, Lin Y, Melhorn SJ, Krause EG, Woods SC, Yanagimachi R, Sakai RR, Tamashiro KLK. Glucose parameters are altered in mouse offspring produced by assisted reproductive technologies and somatic cell nuclear transfer. Biology of Reproduction 2010;83:220-227.

Shansky RM. Are hormones a “female problem” for animal research? Science 2019;364:825826.

Skinner BF. The behavior of organisms. 1938. Appleton-Century-Crofts, New York, NY.

Skinner BF. Science and human behavior. 1953. Macmillan, New York, NY.

Steckler T, Drinkenburgh WH, Sahgal A, Aggleton, JP. Recognition memory in rats-I. Concepts and classification. Progress in Neurobiology 1998;54:289-311.

Stein P, Schultz RM. ICSI in the mouse. Methods in Enzymology 2010;476:251-262.

Sturdy CB, Nicoladis E. How much of language acquisition does operant conditioning explain? Frontiers in Psychology 2017;31,1-5.

Szöke H, Bókkon I, Kapócs G, Vagedes J, Saahs C, Mérey A, Kovács Z. Assisted reproductive technology: Stress-related epigenetic and neurodevelopmental risk? Activitas Nervosa Superior 2018;60:95-106.

Thompson RF, Spencer WA. Habituation: A model phenomenon for the study of neuronal substrates of behavior. Psychological Review 1966;73:16-43. 
787 Thorndike EL. Animal intelligence: Experimental studies. 1911. Macmillan, New York, NY.

788 Yuan S, Stratton, CJ, Bao, J, Zheng, H, Bhetwal, BP, Yanagimachi, R, Yan W. Spata6 is required for normal assembly of the sperm connecting piece and tight head-tail America 2015;112:E430-439. 


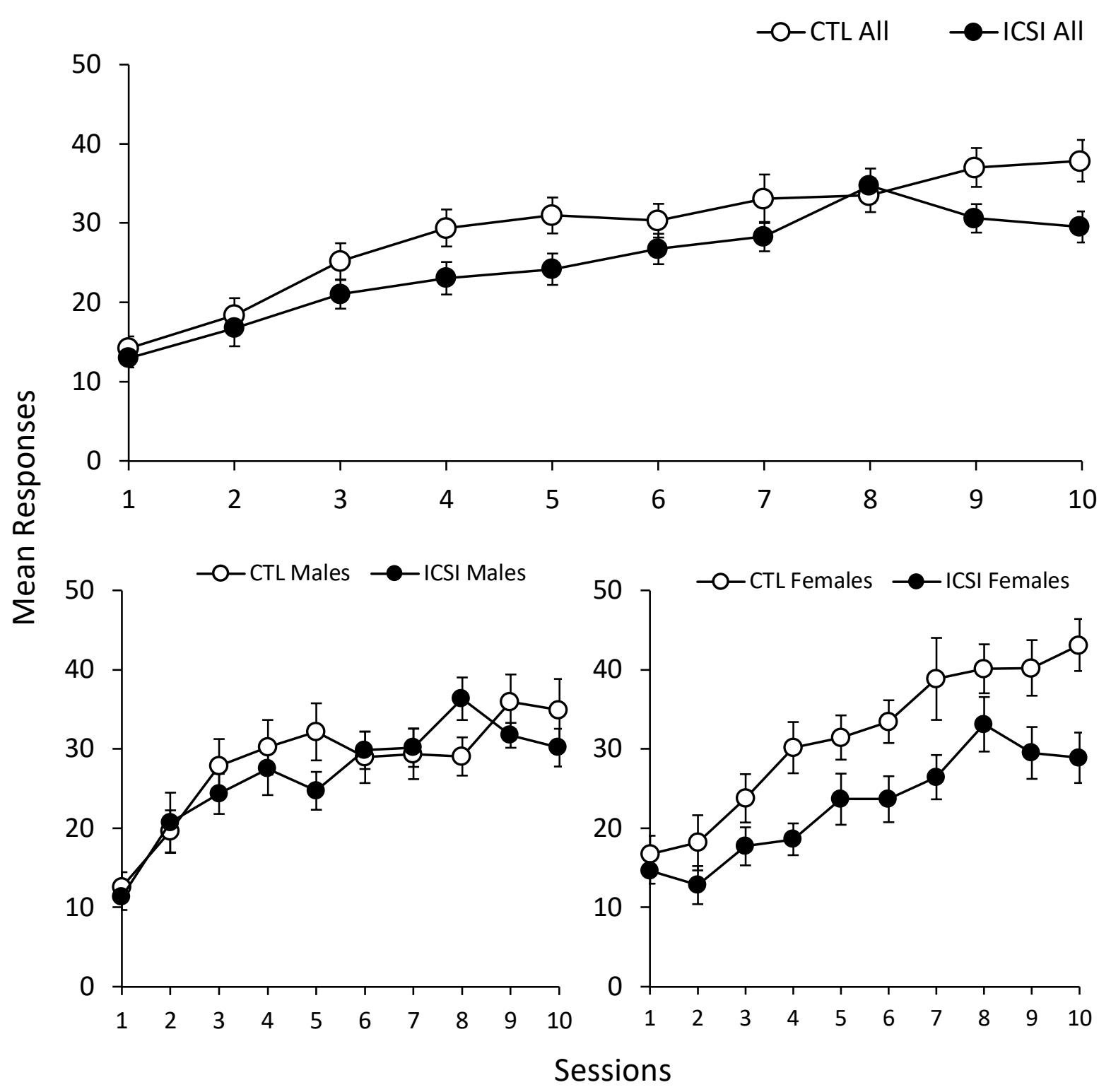

Figure 1. Mean responses per session (+/- standard error of the mean, SEM) during nose poke

794 acquisition sessions for all ICSI and CTL mice (top) and separated by ICSI and CTL males and 795 females (bottom). 


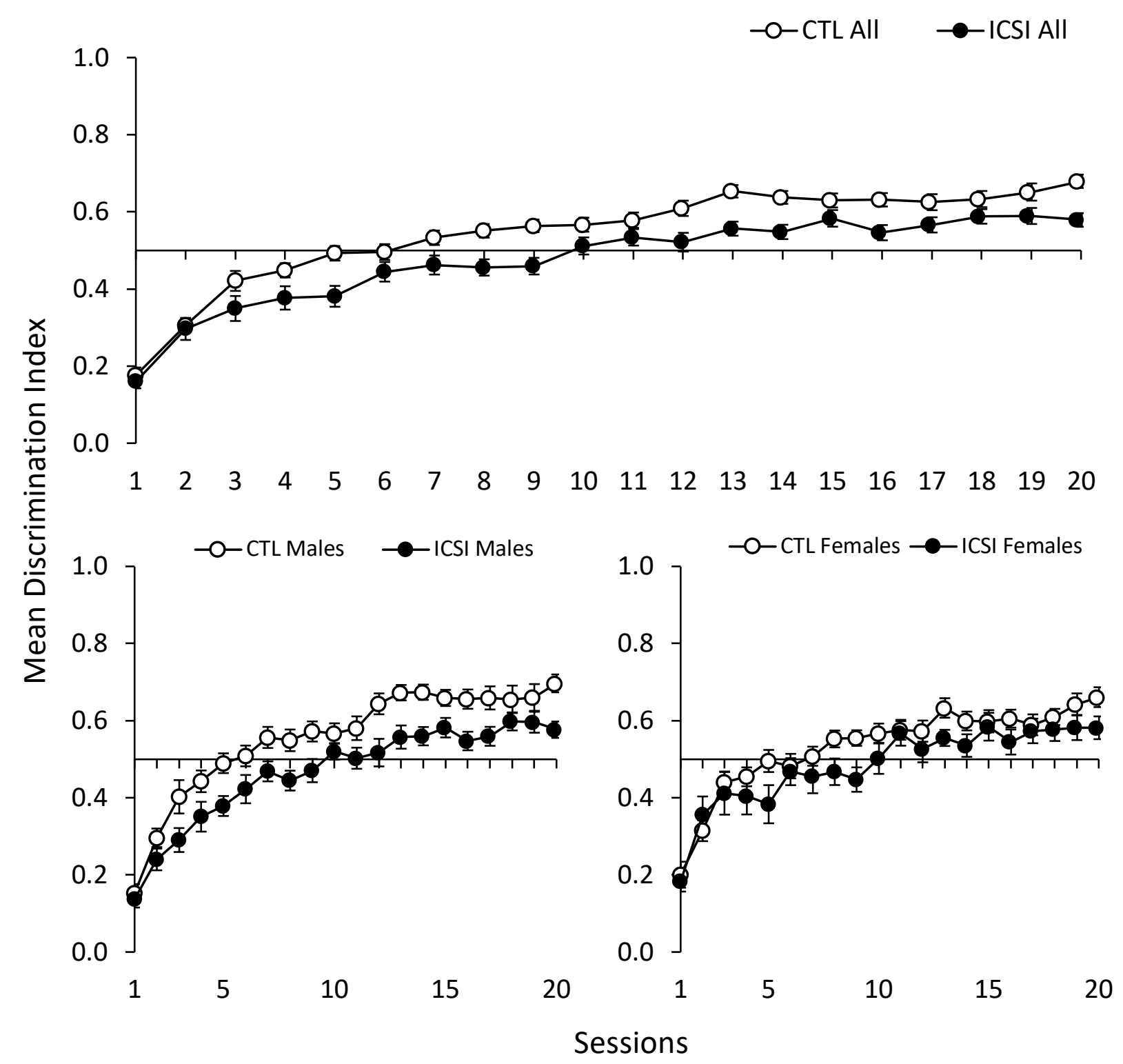

798 Figure 2. Mean discrimination index scores (+/- SEM) during switching discrimination task

799 (SDT) sessions for all ICSI and CTL mice (top) and separated by ICSI and CTL males and 800 females (bottom). 


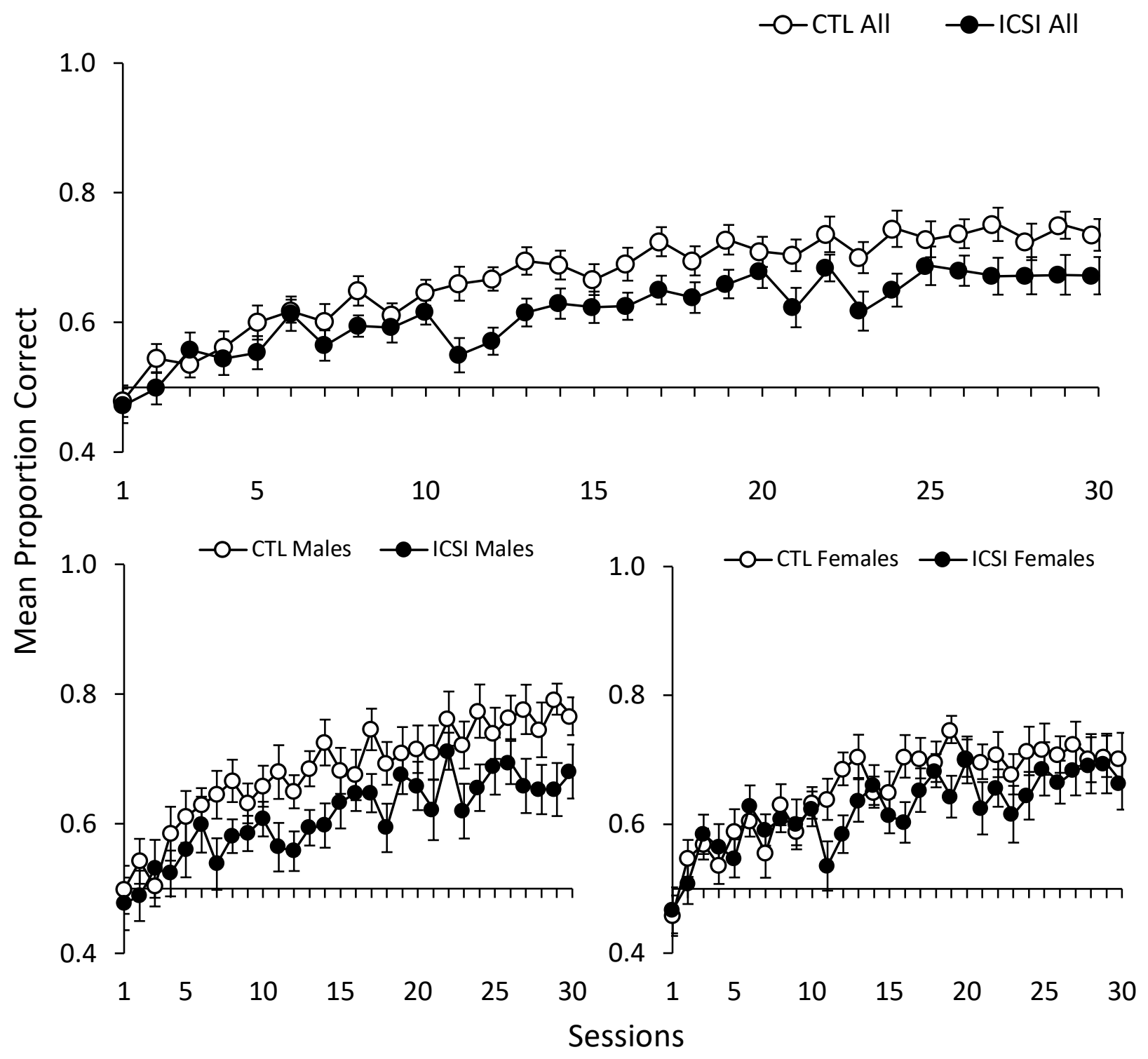

802

803 Figure 3. Mean proportion of correct trials (+/- SEM) in delayed-non-matching-to-position

804 (DNMTP) sessions for all ICSI and CTL mice (top) and separated by ICSI and CTL males and 805 females (bottom). 


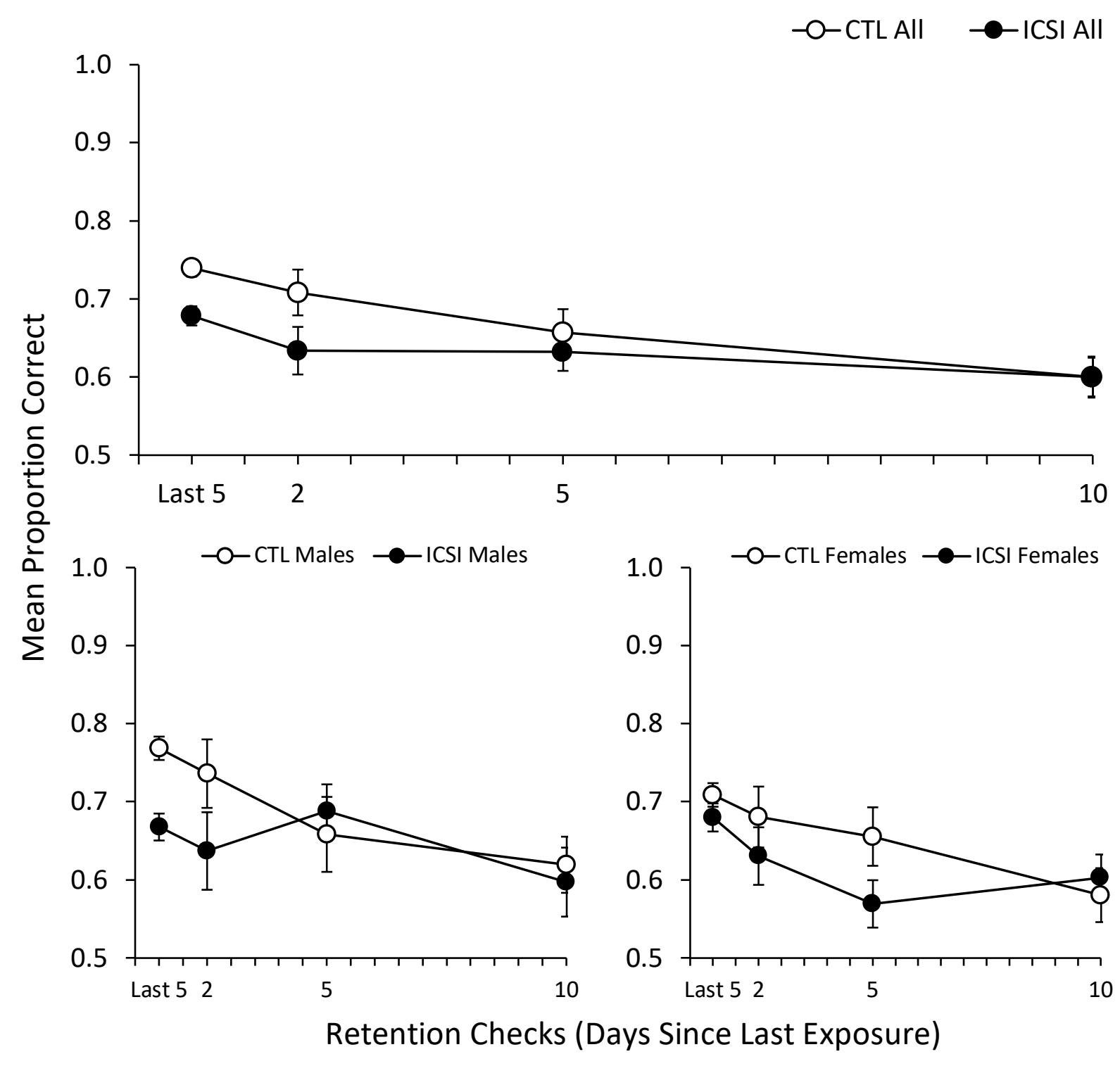

808 Figure 4. Mean proportion of correct trials (+/- SEM) in DNMTP retention checks for all ICSI

809 and CTL mice (top) and separated by ICSI and CTL males and females (bottom). The leftmost

810 data point represents the mean proportion correct by each group in the last five DNMTP training 811 sessions. 


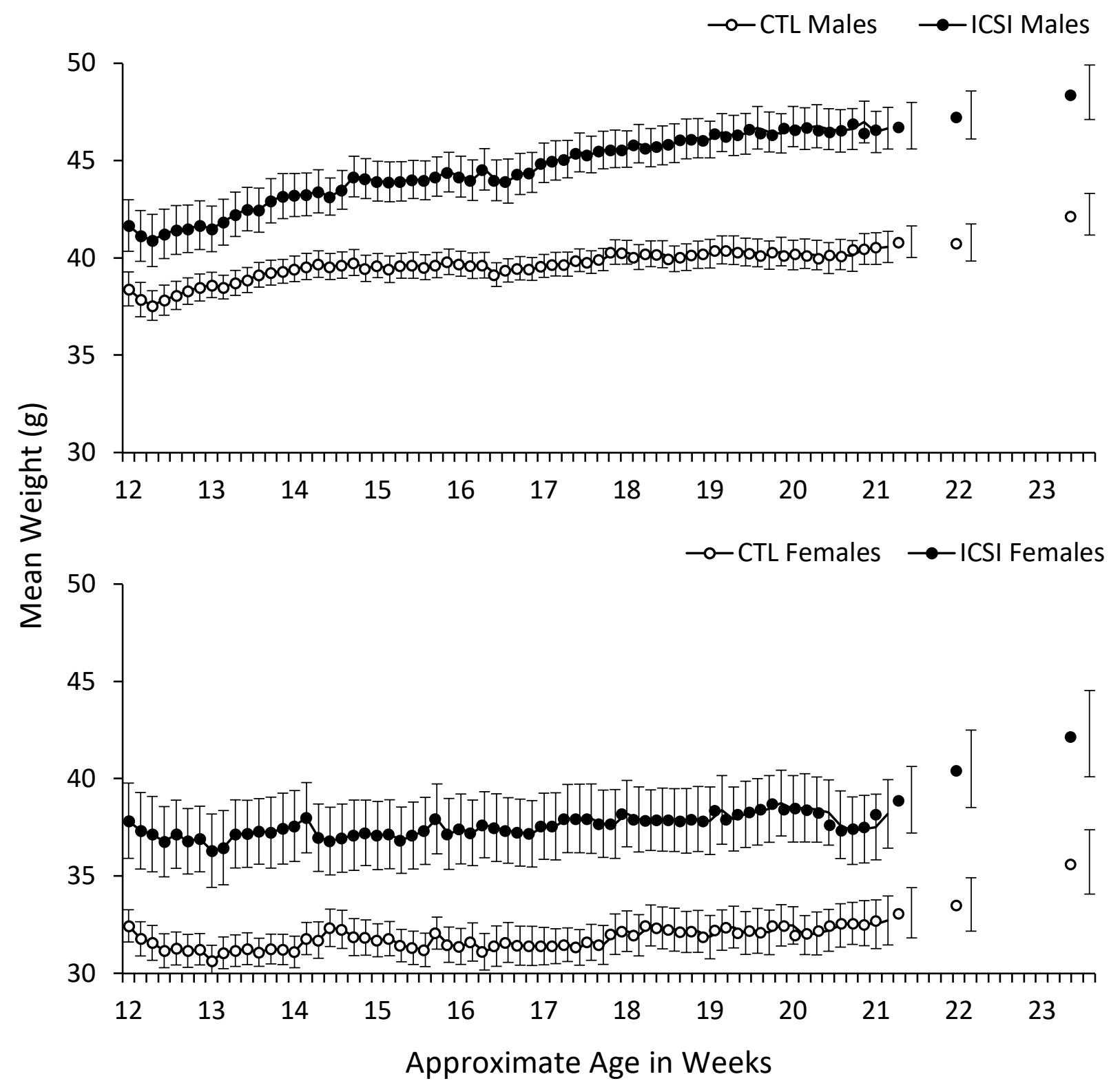

815 Figure 5. Mean daily weights in grams (+/- SEM) for ICSI and CTL males (top) and females

816 (bottom) from the first session of magazine training to the final retention check. The $\mathrm{x}$-axis

817 shows the approximate ages of the mice in weeks. Weights were taken daily prior to sessions

818 following a 14-h period of food deprivation. The gaps in the data series during weeks 21-23 were

819 days between retention checks where mice were not weighed and had continuous free access to

820 food. 


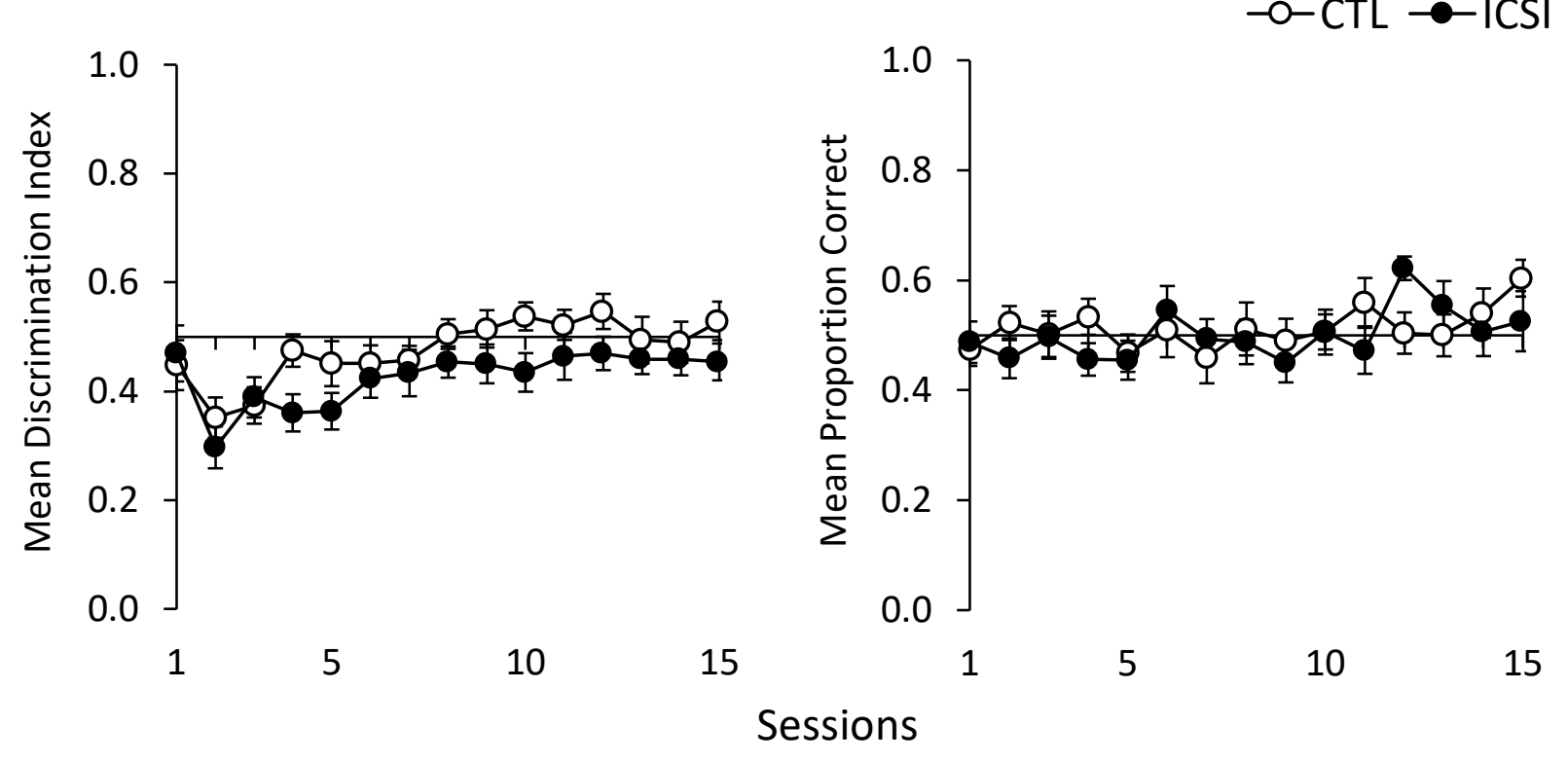

Figure 6. Mean discrimination index during SDT (left) and mean proportion of correct trials

823 during DNMTP (right) follow-up assessments conducted when mice were 52-56 weeks of age.

824 Error bars represent +/- SEM. 


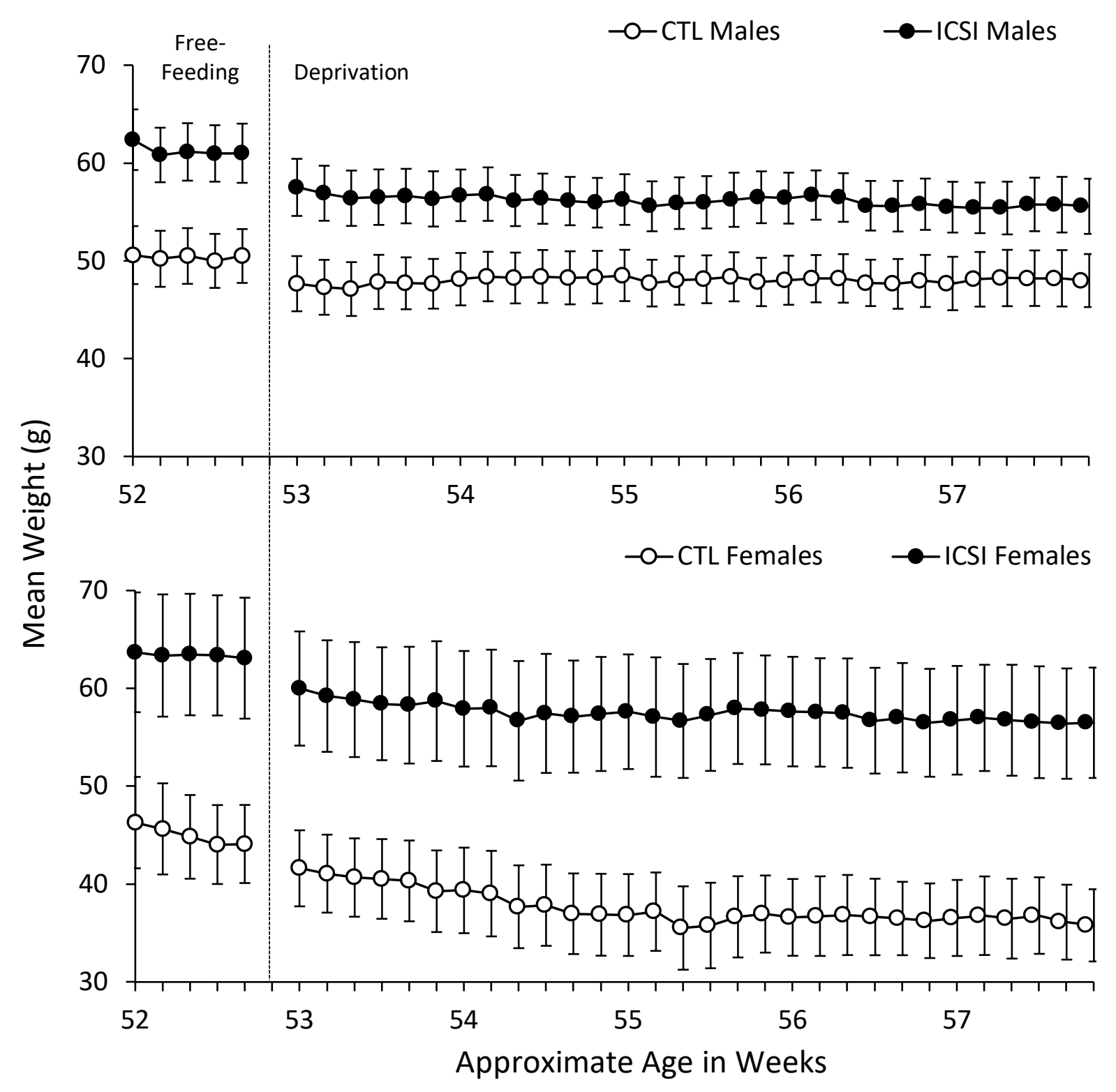

827 Figure 7. Mean daily weights in grams (+/- SEM) for ICSI and CTL males (top) and females

828 (bottom) five days prior to and during the follow-up assessments. Weights to the left of the phase

829 change line were taken daily while mice had free access to food. Weights to the right of the line were taken daily prior to sessions following a 14-h period of food deprivation. 